
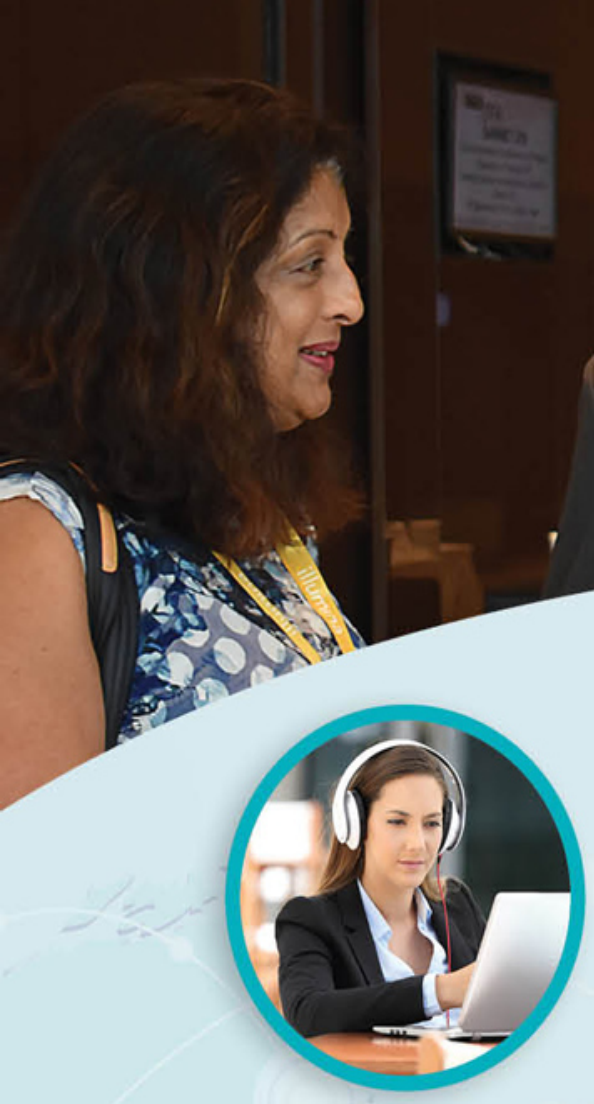

Connect with experts and peers through Special Interest Groups, a searchable member directory, and interactive events.

Stay informed with online access to Prenatal Diagnosis journal, Global Updates e-newsletter, and position statements.
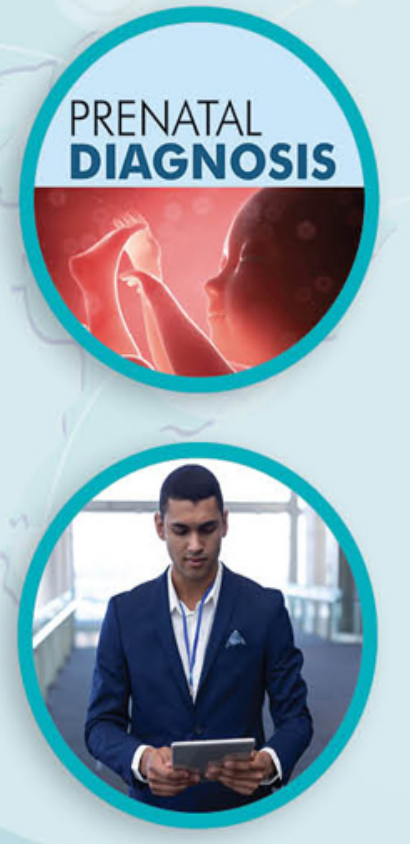

Learn through webinars, the International Conference on Prenatal Diagnosis and Therapy, interviews with experts, and recorded talks.

Professional membership is available for medical and allied health professionals.

Trainee membership is complimentary and includes access to Early Career Resources.

Visit www.ispdhome.org for details.

(3⿻) 1500 for Prenatal Diagnosis Building Global Partnerships in Genetics and Fetal Care

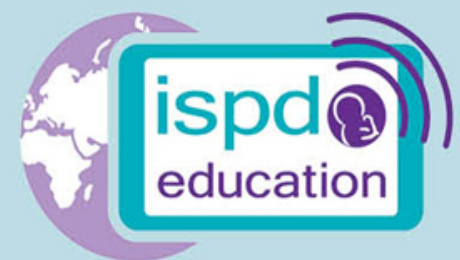

ISPD is a global society of professionals and trainees from disciplines such as prenatal and perinatal care, medical genetics, basic and translational science, genetic counseling, laboratory management, fetal therapy, ultrasonography, and ethics. Become a member today at www.ispdhome.org/join.

f $\mathbf{y}$ in www.ispdhome.org info@ispdhome.org +1434.979.4773 


\section{Fetally-injected drugs for immobilization and analgesia do not modify fetal brain development in a rabbit model}

Short title: Brain development and fetal medication

Wordcount abstract: 202

Wordcount main text: 2852

Tabes: 1

Figures: 4

Lennart Van der Veeken ${ }^{1,2}$, Annalisa Inversetti ${ }^{1}$, Angela Galgano ${ }^{1}$, Tom Bleeser ${ }^{3,4}$, Ioannis Papastefanou ${ }^{5}$, Johannes van de Merwe ${ }^{1,2}$, Steffen Rex $^{3,4}$, Jan Deprest ${ }^{1,2,5}$

${ }^{1}$ Department of Development and Regeneration, Cluster Woman and Child, Group

Biomedical Sciences, KU Leuven, Belgium

${ }^{2}$ Clinical Department Obstetrics and Gynaecology, University Hospitals Leuven, Leuven,

Belgium

${ }^{3}$ Department of Anesthesiology, University Hospitals Leuven, Leuven, Belgium

${ }^{4}$ Department of Cardiovascular Sciences, Group Biomedical Sciences, KU Leuven, Belgium

${ }^{5}$ Fetal Medicine Clinic, Monis Petraki 4, Kolonaki, 11521, Athens, Greece

${ }^{6}$ Institute for Women's Health, University College London, London, UK

Orcid:

- Lennart Van der Veeken: 0000-0002-6551-661X

- Annalisa Inversetti: 0000-0001-8655-9427

- Johannes van der Merwe: 0000-0002-1381-4033

- $\quad$ Steffen Rex: 0000-0002-3536-9321

- Jan Deprest: 0000-0002-4920-945X

Corresponding author: Jan Deprest, MD, PhD, FRCOG: Herestraat 49, 3000 Leuven, Belgium;

jan.deprest@uzleuven.be

This article has been accepted for publication and undergone full peer review but has not been through the copyediting, typesetting, pagination and proofreading process, which may lead to differences between this version and the Version of Record. Please cite this article as doi: 10.1002/pd.5954.

This article is protected by copyright. All rights reserved. 
Disclosure statement: The authors report no conflicts of interest

Funding: LvdV and JvdM are funded by the Erasmus + Programme of the European Union (Framework Agreement number: 2013-0040). This publication reflects the views only of the authors, and the Commission cannot be held responsible for any use which may be made of the information contained therein. Fetal surgery research is also funded by the Wellcome Trust (WT101957) and Engineering and Physical Sciences Research Council (ESPRC) (NS/A000027/1). JD is funded by the Great Ormond Street Hospital Charity Fund.

Bulleted statements:

A. What's already known about this topic?

- During fetal surgery, fetuses receive medication (atropine-fentanyl-curare) to prevent fetal pain, movement and bradycardia.

- Maternal anesthesia can cause impaired brain development

- There are no reports on neurodevelopmental effects of direct fetal anesthesia

B. What does this study add?

- This is the first study investigating the effects of fetal medication

- Many centers still rely on the transplacental transfer of maternal anesthesia to achieve fetal analgesia. Based on these findings, a direct fetal injection with medication would be preferred.

Ethics approval: These experiments were approved on 18/04/2018 by the Ethics Committee for Animal Experimentation of the Faculty of Medicine of the Katholieke Universiteit Leuven (LA1210191) (P078/2017).

Data sharing: The data that support the findings of this study are available from the corresponding author upon reasonable request.

This article is protected by copyright. All rights reserved. 
Keywords: Fetal anesthesia, fetal analgesia, fetal immobilization, fetal surgery, brain development, neurobehavior, rabbit

This article is protected by copyright. All rights reserved. 


\section{Abstract}

Objective: During fetal surgery, fetuses receive medication (atropine-fentanyl-curare) to prevent fetal pain, movement and bradycardia. Although essential there has been no detailed review of potential side effects. Herein we aimed to assess the effects of this medication cocktail on fetal brain development in a rabbit model.

Methods: Pregnant does underwent laparotomy at $28 \mathrm{~d}$ of gestation. Two pups of each horn were randomized to an ultrasound guided injection with medication (atropine-cisatracurium-fentanyl, as clinically used) or saline (sham). The third pup was used as control. At term, does were delivered by cesarean. Outcome measures were neonatal biometry, neuromotoric functioning and neuro-histology (neuron density, synaptic density and proliferation).

Results: Maternal vital parameters remained stable during surgery. Fetal heart rates did not differ before and after injection, and were comparable for the three groups. At birth, neonatal body weights and brain-to-body weight ratios were also comparable. Both motor and sensory neurobehavioral scores were comparable. There were no differences in neuron density or proliferation. Sham pups, had a lower synaptic density in the hippocampus as compared to the medication group, however there was no difference in the other brain areas.

Conclusion: In the rabbit model, fetal medication does not appear to lead to short-term neurocognitive effects.

This article is protected by copyright. All rights reserved. 


\section{Introduction}

Fetal surgery has rapidly progressed during the last decades, both in terms of indications for interventions as well as advances in operative techniques. Selected fetuses now benefit from prenatal surgery with improved short- and long-term outcomes ${ }^{1-3}$. The time point in gestation for these procedures to be performed, depends on the indication, though typically gestational age (GA) ranges from 16 weeks to the time point at which ex utero management is preferred.

It remains uncertain and debated from what gestational age and to which degree a fetus is capable of experiencing pain. Therefore it is conservative to presume that fetuses are capable of experiencing pain from the second half of pregnancy ${ }^{4,5}$. Moreover, neonatal repetitive noxious stimuli alter normal neurodevelopment in rat pups ${ }^{6}$. Therefore, providing adequate fetal analgesia is important ${ }^{7}$. Fetal analgesia is frequently combined with a muscle relaxant to prevent sudden fetal movements which could be harmful during the procedure. To counteract possible bradycardia caused by fetal pain or during fetal laryngoscopy, some centers add atropine to the fetal medication. Therefore, fetuses are being administered a combination of fentanyl, a muscle relaxant and atropine either by intravenous (IV) (umbilical or hepatic vein) or intramuscular (IM) injection.

Multiple animal studies demonstrated that lengthy or repetitive use of maternal anesthesia can cause impaired brain development ${ }^{8}$. These observations led to an FDA safety communication for the use of general anesthetics in pregnant women ${ }^{9}$. We previously demonstrated in the rabbit model that a single two-hour maternal surgery caused transient negative neurobehavior and brain histopathological changes in the fetus ${ }^{10}$. The use of maternal anesthetic agents in that study was associated with a lower neuron density, lower synaptic density and decreased the proliferation rates in multiple brain regions. The pups of exposed does had a worse neurobehavioral outcome at birth. However unlike maternal anesthetic medication given during surgery, which has been well investigated, the neurodevelopmental effects of drugs directly administered to the fetus have not yet been investigated. Due to the many confounding factors and because of ethical reasons, it is (nearly) impossible to investigate the effect of fetal medication in humans. Therefore we translated this to our rabbit

This article is protected by copyright. All rights reserved. 
neurodevelopment model and herein we aimed to measure the effect of fetal medication during surgery under general anesthesia.

We opted for the rabbit model because similar to humans, the rabbit is a perinatal brain developer ${ }^{11}$ and many evaluations have been standardized to investigate perinatal insults ${ }^{10}$.

This article is protected by copyright. All rights reserved. 


\section{Material and Methods}

Time-mated rabbits (hybrid of Vlaamse Reus and New-Zealand White) were housed in individual cages at $21^{\circ} \mathrm{C}, 42 \%$ humidity with a $12 \mathrm{~h}$ day-night cycle and free access to water and food. Animals were treated according to current guidelines for animal well-being, and experiments were approved by the Ethics Committee for Animal Experimentation of the Faculty of Medicine (P078/2017).

Anesthetic and surgical procedures (Figure 1)

At gestational day 28, pregnant does were anesthetized with sevoflurane as previously described ${ }^{10}$. A median laparotomy $(5 \mathrm{~cm})$ was made to partially expose the uterus and allow micro-ultrasound Doppler (Vevo 2100 Imaging System, Fujifilm VisualSonics Inc., Toronto, ON, Canada). With minimal manipulation, the two ovarian-end pups of each horn were randomized to receive an ultrasound guided intrahepatic injection either with $0.1 \mathrm{~mL}$ fetal medication or $0.1 \mathrm{~mL}$ saline (sham). The third ovarian end pups from each horn were used as controls. Fetal medication was standardized to fentanyl $0.2 \mu \mathrm{g}$, cisatracurium $10 \mu \mathrm{g}$ and atropine $0.2 \mu \mathrm{g}$. This was based on clinical equivalent concentrations (fentanyl $12 \mu \mathrm{g} / \mathrm{kg}$, cisatracurium $0.4 \mathrm{mg} / \mathrm{kg}$ and atropine $13 \mu \mathrm{g} / \mathrm{kg}$ ) and available average weights of pups of similar gestational age from a previous study ${ }^{12}$ ). The fetal heart rate was measured with ultrasound before and 10 minutes after injection. Maternal anesthesia was standardized to $1 \mathrm{~h}$ of sevoflurane maintenance.

Once awake, animals were returned to their cage for recovery. During the remainder of the pregnancy, rabbits were daily monitored using the Rabbit Grimace Scale. With this scale rabbits are observed for a couple of minutes and five action units are scored: orbital tightening, cheek flattening, nostril shape, whisker change \& position and ear shape \& position ${ }^{13}$. Each item is scored as either 0 (not present), 1 (moderately present) or 2 (obviously present).

\section{Delivery}

Caesarean was performed at postconceptional day 31 (term). After sedation with IM ketamine (15 $\mathrm{mg} / \mathrm{kg}$, ketamine Nimatek; Eurovet Animal Health BV, Bladel, The Netherlands) and medetomidine (25 $\mathrm{mg} / \mathrm{kg}$, Domintor, Orion Pharma, Aartselaar, Belgium), the doe was placed in the supine position, and

This article is protected by copyright. All rights reserved. 
$2 \%$ lidocaine local anesthesia was injected to allow median laparotomy. The bicornuate uterus was exposed and all pups were extracted through hysterotomy. Following delivery, the doe was euthanized with a mixture of $200 \mathrm{mg}$ embutramide, $50 \mathrm{mg}$ mebezonium, and $5 \mathrm{mg}$ tetracain hydrochloride (IV bolus of $1 \mathrm{~mL}$ T61; Intervet International BV, Boxmeer, The Netherlands).

After birth the pups were dried, stimulated and kept in an incubator (TLC-50 Advance, Brinsea Products, Weston Super Mare, UK) set on $32^{\circ} \mathrm{C}$ and $55 \%$ humidity. Litters were separately housed in plastic boxes $(20 \times 25 \mathrm{~cm})$ and pups were numbered according to their position in utero. Pups were gavage fed twice daily with Milk Replacer (FoxValley 30/50, Lakemoor Illinois, US) supplemented with probiotics and colostrum (Col-o-cat, Sanobest, 's Hertogenbosch, Netherlands) at $150 \mathrm{~mL} / \mathrm{kg} / \mathrm{feeding}$. At each feeding, the bladder was emptied by genital stimulation.

\section{Neurobehavioral assessments}

On postnatal day one, all pups underwent neurobehavioral assessment, as used in previous studies 10,12. All tests were performed out of the incubator, and for each pup individually. For neuromotor evaluation, an open field test was performed on a designated $30 \times 30 \mathrm{~cm}$ area divided in quadrants over a time period of 90 seconds. Each animal was left free to ambulate. The following items were scored: locomotion; motor activity of head, for and hind limbs; limb tone. The sum of these scores represented the total motor score, that was calculated out of a maximum of 26 points. Sensory testing included: soft sensation, sucking and swallowing, head turning, surface righting reflex, odor aversion and pain sensation. The maximum sensory score attainable was 20 points.

All assessments (performed by blinded investigators) were recorded and videos were scored $a$ posteriori by two observers blinded to group assignment.

\section{Neuropathological assessments}

After neurobehavioral testing rabbits were anesthetized with IM ketamine $(35 \mathrm{mg} / \mathrm{kg})$ and xylazin (6 $\mathrm{mg} / \mathrm{kg})$ and were perfused transcardially with $0.9 \%$ saline and heparin $(100 \mathrm{IU} / \mathrm{mL})$ followed by perfusion fixation with $4 \%$ formaldehyde. The brain was removed to be immersion fixed in $4 \%$

This article is protected by copyright. All rights reserved. 
formaldehyde. Whole brain volumes, including cerebellum and brainstem, were determined using the fluid displacement method. After 72 hours, brains were paraffin embedded and subsequently serially sectioned at $4 \mu \mathrm{m}$ from anterior to posterior, starting from the prefrontal cortex. Sets of 3 coronal sections were taken every $100 \mu \mathrm{m}$, at two levels for further staining and analysis. The first level started at the start of the medial septal nucleus and the second level at the beginning of the hippocampal formation. Primary antibodies used in these experiments included: mouse monoclonal anti-NeuN antibody (MAB377, Millipore, Billerica, MA, USA); mouse monoclonal anti-human Ki67 (M724001-2; Agilent, Diegem, Belgium); mouse anti-synaptophysin monoclonal antibody (Sy38, ab8049; Abcam, Cambridge, UK). The secondary antibody was Alexa Fluor ${ }^{\circledR} 488$ goat anti-mouse conjugate (Invitrogen, Thermo Fisher Scientific, Waltham, Massachusetts, USA). Sections were counterstained with Hoechst 33342 (Sigma-Aldrich, Bornem, Belgium).

\section{Imaging Acquisition and Quantification}

Histological slides were digitized using the Zeiss AxioScan Z1 imaging platform (AxioScan ${ }^{\circledR}$ Slide Scanner, Carl Zeiss Microlmaging GmbH, Munich, Germany), using a 20x Plan Apochromat objective coupled to a 3 Chip CCD Camera (Hamamatsu Photonics, Japan). All focusing and field of view assembly was handled by Carl Zeiss Zen software (Carl Zeiss Microlmaging GmbH, Munich, Germany), which is integrated with the AxioScan device.

For neuronal densities, regions of interest (ROI) were selected on three consecutive slides. In each ROI, five different $100 \times 100 \mu \mathrm{m}$ squares were selected at low magnification so individual cells were not visible to avoid bias. In these squares individual neurons were manually counted.

For quantification of the Ki-67 positive cells quantification profiles on the digitized whole slide images were done using QuPath software ${ }^{14}$ by utilizing the fast cell counting and positive cell detection functions. Herein the whole region of interest was annotated, quantification was done, and positive cell counts were expressed as percentage of positive cells per total cells detected.

For the mean fluorescence intensity, quantification of the synaptophysin stain (Sy38) open source Fiji software (ImageJ) (http://fiji.sc/Fiji) ${ }^{15}$ was used.

This article is protected by copyright. All rights reserved. 


\section{Statistics}

The study was powered to detect a $15 \%$ difference in neuron density (based on findings in a previous experiment on maternal surgery during pregnancy). Data was analyzed using $\mathrm{R}$ (Foundation for Statistical Computing, Vienna, Austria). Data distribution was checked and confirmed for normality by the Shapiro Wilk test and normality plots. Comparisons of the outcomes between the treatment groups was carried out with Analysis of Variance (ANOVA) assuming equal variances. We used linear mixed models to account for the potential clustering effect in our data. Post hoc comparisons were done with Students t-test with Bonferroni-correction for multiple testing. A p-value $<0.05$ was considered significant. All data are presented as means with standard deviation and individual data points are plotted.

This article is protected by copyright. All rights reserved. 


\section{Results}

Eight pregnant does underwent surgery and had live born pups. During the first surgery, maternal vital signs remained stable and were within the normal range except for two rabbits that had hypotension throughout surgery ${ }^{16}$. Immediately after intubation, the does had a slightly lower $\mathrm{pH}(7.32 \pm 0.08$; $\mathrm{p}=0.02$ ) compared to normal, however, $\mathrm{pCO}_{2}$ and lactate levels remained within their normal range. At the end of surgery, $\mathrm{pH}$ values were comparable and within the normal range $(7.40 \pm 0.04 ; \mathrm{p}=0.6)$ (Table $1)$.

Fetal heart rates of the three groups were comparable before injection (192 \pm 47 control; $189 \pm 40$ sham; $182 \pm 49$ medication; $p=0.4$ ). Following injection, the average heart rate did not change, both in the sham group $(p=0.5)$ as in medication group $(p=0.2)$ (Supplement Figure 1$)$. Also there were no differences between the three groups at the second time point.

Survival till birth was comparable ( $79 \%$ control; $80 \%$ sham; $63 \%$ medication $\mathrm{p}=0.5)$. Therefore, we eventually harvested 10 control, 12 sham and 9 medicated pups, with comparable body, brain weights and brain-to-body weight ratios (Supplement Figure 1).

The total motor scores $(p=0.4)$ and total sensory scores $(p=0.2)$ were not different, even not for any of the sub-scores (data not shown) (Figure 2).

Neuron densities were comparable for all studied brain regions (Figure 3). There were neither any differences in proliferation rates (Supplement Figure 2). There was a slightly higher synaptic density in the hippocampus of animals injected with medication as compared to the two other groups (1641 \pm 437 control; $1695 \pm 330$ sham; $2324 \pm 314$ medication; $p=0.004$ ). In the other regions no differences could be detected between the groups (Figure 4).

This article is protected by copyright. All rights reserved. 


\section{Discussion}

This is to our knowledge the first study exploring the effects of widely used fetal injected atropinefentanyl-curare on fetal brain development. Whereas in a previous experiment in the same model, we demonstrated measurable neonatal, yet transient functional and morphological neurologic changes following general anesthesia, herein, no effect of direct fetal injected fentanyl, cisatracurium and atropine was demonstrated. There was only a higher synaptic density in the hippocampus of pups injected with the fetal medication cocktail, without measurable functional impact in the early neonatal period.

To study the effects of the fetal medication cocktail, we resorted to the rabbit model. The rabbit model is a very suitable model to investigate the impact of potentially noxious events on fetal brain development. Rabbits are so-called perinatal brain developers, which means that their brain mainly develops in the third trimester and neonatal period, as is the case in humans ${ }^{11}$. We injected fetal medication on gestational day 28 which, in terms of brain development, corresponds to gestational week 24-28 in humans, the period when fetal surgery is typically performed ${ }^{17}$. The early third trimester also marks the beginning of the brain growth spurt both in rabbits as well as humans, which makes the brain most sensitive to external disruptive factors ${ }^{18,19}$.

The mix we used consisted of fentanyl, cisatracurium and atropine in clinically equivalent doses ${ }^{20}$. Atropine is used by several fetal surgery centers to prevent fetal bradycardia which can be triggered by fetal pain or e.g. by laryngoscopy during Fetosopic Endoluminal Tracheal Occlsuion (FETO) procedures $^{21,22}$. Whether the use of atropine is essential is unknown. To our knowledge there is nothing reported on the use and postnatal effects of atropine administered to fetuses. Also, in this model, it is uncertain whether one can properly study the fetal effects, as up to $70 \%$ of New Zealand white rabbits have an esterase which immediately degrades atropine, before it has the time to exert any effects ${ }^{23}$. This may not be impossible, as we did not observe the (expected) increase in fetal heart rate.

We could not demonstrate differences between control fetuses or fetuses injected with saline or with fetal medication in terms of growth, neurobehavioral assessment or in neuron density.

This article is protected by copyright. All rights reserved. 
There is no literature on the brain effects of opioids directly given to the fetus. Fisk et al. demonstrated that fentanyl attenuates fetal stress during needling procedures but they did not investigate brain changes $^{24}$. Maternally administered opioids and its metabolites cross the placenta, hence exert effects on the fetus. Both clinical and preclinical studies demonstrated that sustained maternal opioids administration adversely impacts fetal brain development, leading to lower neuroproliferation rate, lower neuron density and functional impairment ${ }^{25-28}$. Whereas these studies investigated chronic maternal opioid administration, single maternal exposure studies are scarce. Rizzi et al. administered a single dose of fentanyl to pregnant guinea pigs for anesthetic purposes and did not observe any effect. Though the guinea pig may not be the ideal model to study perinatal brain development, those observations are in line with ours, i.e. no difference in neuron density following a single administration ${ }^{11,29}$.

The effect of cistracurium or other curariform products on the developing brain is also poorly studied. Fodale et al. suggested a potential neuroprotective effect of laudanosine on the fetal brain based on preclinical data in rats and in vitro studies ${ }^{30}$. Laudanosine is a metabolite of cisatracurium and a nicotine acetylcholine subtype $\alpha 4 \beta 2$ receptor agonist ${ }^{31}$. Activation of this receptor has been shown to provide neuroprotection against NMDA ( $\mathrm{N}$-methyl-D-aspartate) excitotoxicity in a neonatal rat mode $^{32}$. In our previous experiment we hypothesized that excitotoxicity was a potential mechanism underlying adverse neurologic effects. Therefore the protective effect of laudanosine against the excitotoxicity of general anesthesia could explain the higher synaptic density measured in the hippocampus. However in that case we would also expect to see a higher proliferation rate and higher neuron density, yet we only observed a higher synaptic density in the hippocampus. Therefore it is also possible that this was an incidental finding.

This study has some limitations. First this experiment includes a maternal laparotomy under general anesthesia, which is not always the case in fetal surgical procedures. To control for this, we used internal controls exposed to the same drugs. Secondly, we administered fetal medication in the liver whereas in humans, it is given either IM or IV. In rabbits, due to the small size of the veins, it is in our

This article is protected by copyright. All rights reserved. 
hands impossible to administer fetal medication IV. Also an IM injection is difficult to control due to the small limb size and the fetal movements. When medication was given intrahepatically, ultrasound imaging enabled us to keep the injected fluid on screen until the uptake was completed. This was in all cases accomplished within $30 \mathrm{sec}$. Thirdly, since we did not see an effect on day 1, we did not perform long term follow up of the rabbits, which does not exclude long term effects. Lastly, although the rabbit is a suitable model for perinatal insults, the rabbit brain is far less complex than the human brain. In addition, there are differences in equipotency of drugs between adults and children and between humans and rabbits. Although we used a clinical equivalent dose of medication we cannot be $100 \%$ certain that this has the same potency in rabbit fetuses.

Our study also has some strengths. First the choice of the rabbit model is a very good model for brain development. The rabbit is a perinatal brain developer, like humans, unlike rodents (postnatal), sheep or even monkeys (prenatal) ${ }^{11}$. Therefore, brain growth in rabbits mimics the human situation. This allowed us to perform the intervention at a developmental stage which corresponds to the timeframe when fetal surgeries happen. Secondly, the neonatal rabbit model has already frequently been used to study perinatal brain insults ${ }^{33-37}$. Thirdly, the large litter size allowed us to compare treatments within each mother. Lastly, using the same protocol of maternal anesthesia for all the does, we were able to dissect out the singular effect of fetal injected atropine-fentanyl-curare on brain development, which is hard to analyze in the human setting due to the many confounding factors.

\section{Conclusion}

In the rabbit model, additional administration of fetal injected atropine-fentanyl-curare does not lead to measurable short-term neurocognitive effects. Many centers still rely on general maternal anesthesia to achieve fetal analgesia and immobilization ${ }^{38}$. Based on these findings and our previous findings on maternal anesthesia in the rabbit model, a direct fetal injection with medication would be preferred to achieve fetal analgesia.

This article is protected by copyright. All rights reserved. 


\section{REFERENCES}

1. Adzick NS, Thom EA, Spong $\mathrm{CY}$, et al. A randomized trial of prenatal versus postnatal repair of myelomeningocele. N Engl J Med 2011; 364(11): 993-1004.

2. Dekoninck P, Gratacos E, Van Mieghem T, et al. Results of fetal endoscopic tracheal occlusion for congenital diaphragmatic hernia and the set up of the randomized controlled TOTAL trial. Early Hum Dev 2011; 87(9): 619-24.

3. Senat MV, Deprest J, Boulvain M, Paupe A, Winer N, Ville Y. Endoscopic laser surgery versus serial amnioreduction for severe twin-to-twin transfusion syndrome. N Engl J Med 2004; 351(2): 13644.

4. Van de Velde M, Jani J, De Buck F, Deprest J. Fetal pain perception and pain management. Semin Fetal Neonatal Med 2006; 11(4): 232-6.

5. $\quad$ Bellieni CV. New insights into fetal pain. Semin Fetal Neonatal Med 2019; 24(4): 101001.

6. Anand KJ, Coskun V, Thrivikraman KV, Nemeroff CB, Plotsky PM. Long-term behavioral effects of repetitive pain in neonatal rat pups. Physiology \& behavior 1999; 66(4): 627-37.

7. Deprest J, Toelen J, Debyser Z, et al. The fetal patient -- ethical aspects of fetal therapy. Facts, views \& vision in ObGyn 2011; 3(3): 221-7.

8. Palanisamy A. Maternal anesthesia and fetal neurodevelopment. Int J Obstet Anesth 2012; 21(2): 152-62.

9. Announcement. FDA review results in new warnings about using general anesthetics and sedation drugs in young children and pregnant women. . 2016.

10. Van der Veeken L, Van der Merwe J, Devroe S, et al. Maternal surgery during pregnancy has a transient adverse effect on the developing fetal rabbit brain. Am J Obstet Gynecol 2019.

11. Dobbing J, Sands J. Comparative aspects of the brain growth spurt. Early Hum Dev 1979; 3(1): 79-83.

12. van der Merwe J, van der Veeken L, Ferraris S, et al. Early neuropathological and neurobehavioral consequences of preterm birth in a rabbit model. Scientific reports 2019; 9(1): 3506.

13. Hampshire V, Robertson S. Using the facial grimace scale to evaluate rabbit wellness in postprocedural monitoring. Lab animal 2015; 44(7): 259-60.

14. Bankhead P, Loughrey MB, Fernandez JA, et al. QuPath: Open source software for digital pathology image analysis. Scientific reports 2017; 7(1): 16878.

15. Schindelin J, Arganda-Carreras I, Frise E, et al. Fiji: an open-source platform for biological-image analysis. Nature methods 2012; 9(7): 676-82.

16. Bleeser T, Van Der Veeken L, Devroe S, Deprest J, Rex S. Arterial blood pressure and arterial blood gas values in conscious pregnant rabbits. Veterinary anaesthesia and analgesia 2020.

17. Clancy B, Finlay BL, Darlington RB, Anand KJ. Extrapolating brain development from experimental species to humans. Neurotoxicology 2007; 28(5): 931-7.

18. Dobbing J, Sands J. Vulnerability of developing brain. IX. The effect of nutritional growth retardation on the timing of the brain growth-spurt. Biology of the neonate 1971; 19(4): 363-78.

19. Dobbing J. Brain, Behavior and Iron in the Infant Diet. London: Springer; 1990.

20. Van de Velde M, De Buck F. Fetal and maternal analgesia/anesthesia for fetal procedures. Fetal Diagn Ther 2012; 31(4): 201-9.

21. Mayorga-Buiza MJ, Marquez-Rivas J, Gomez-Gonzalez E. Can fetus feel pain in the second trimester? Lessons learned from a sentinel event. Childs Nerv Syst 2018; 34(2): 195-6.

22. Shaw CA, Kelleher AA, Gill CP, Murdoch L, Stables RH, Black AE. Comparison of the incidence of complications at induction and emergence in infants receiving oral atropine vs no premedication. Br J Anaesth 2000; 84(2): 174-8.

23. Linn JM, Liebenberg SP. In vivo detection of rabbit atropinesterase. Laboratory animal science 1979; 29(3): 335-7.

24. Fisk NM, Gitau R, Teixeira JM, Giannakoulopoulos X, Cameron AD, Glover VA. Effect of direct fetal opioid analgesia on fetal hormonal and hemodynamic stress response to intrauterine needling. Anesthesiology 2001; 95(4): 828-35.

This article is protected by copyright. All rights reserved. 
25. Azuine RE, Ji Y, Chang HY, et al. Prenatal Risk Factors and Perinatal and Postnatal Outcomes Associated With Maternal Opioid Exposure in an Urban, Low-Income, Multiethnic US Population. JAMA network open 2019; 2(6): e196405.

26. Caritis SN, Panigrahy A. Opioids affect the fetal brain: reframing the detoxification debate. $A m$ J Obstet Gynecol 2019; 221(6): 602-8.

27. Sadraie SH, Kaka GR, Sahraei H, et al. Effects of maternal oral administration of morphine sulfate on developing rat fetal cerebrum: a morphometrical evaluation. Brain research 2008; 1245: 3640.

28. Wu VW, Mo Q, Yabe T, Schwartz JP, Robinson SE. Perinatal opioids reduce striatal nerve growth factor content in rat striatum. Eur J Pharmacol 2001; 414(2-3): 211-4.

29. Rizzi S, Carter LB, Ori C, Jevtovic-Todorovic V. Clinical anesthesia causes permanent damage to the fetal guinea pig brain. Brain Pathol 2008; 18(2): 198-210.

30. Fodale V, Pratico C, Signer MR, Santamaria LB. Perinatal neuroprotection by muscle relaxants against hypoxic-ischemic lesions: is it a possible hypothesis? J Matern Fetal Neonatal Med 2005; 18(2): 133-6.

31. Tassonyi E, Fathi M, Hughes GJ, et al. Cerebrospinal fluid concentrations of atracurium, laudanosine and vecuronium following clinical subarachnoid hemorrhage. Acta anaesthesiologica Scandinavica 2002; 46(10): 1236-41.

32. Laudenbach V, Medja F, Zoli M, et al. Selective activation of central subtypes of the nicotinic acetylcholine receptor has opposite effects on neonatal excitotoxic brain injuries. FASEB journal : official publication of the Federation of American Societies for Experimental Biology 2002; 16(3): 4235.

33. Mitchell RL, Barbano TE, Losken HW, Siegel MI, Mooney MP. Early neuromotor behavior in craniosynostotic rabbits. The Cleft palate-craniofacial journal : official publication of the American Cleft Palate-Craniofacial Association 2003; 40(5): 486-92.

34. Derrick M, Luo NL, Bregman JC, et al. Preterm fetal hypoxia-ischemia causes hypertonia and motor deficits in the neonatal rabbit: a model for human cerebral palsy? J Neurosci 2004; 24(1): 24-34. 35. Drobyshevsky A, Song SK, Gamkrelidze G, et al. Developmental changes in diffusion anisotropy coincide with immature oligodendrocyte progression and maturation of compound action potential. $J$ Neurosci 2005; 25(25): 5988-97.

36. Eixarch $\mathrm{E}$, Batalle $\mathrm{D}$, Illa $\mathrm{M}$, et al. Neonatal neurobehavior and diffusion MRI changes in brain reorganization due to intrauterine growth restriction in a rabbit model. PLoS One 2012; 7(2): e31497. 37. Gram M, Sveinsdottir S, Cinthio M, et al. Extracellular hemoglobin - mediator of inflammation and cell death in the choroid plexus following preterm intraventricular hemorrhage. Journal of neuroinflammation 2014; 11: 200.

38. Noguchi S, Tanaka M, Terui K. The first national survey of anesthesia techniques for fetal therapies in Japan. Journal of anesthesia 2019; 33(6): 665-9.

This article is protected by copyright. All rights reserved. 
Table 1: Maternal parameters measured during surgery. MAP: Mean Arterial Pressure; HR: Heart Rate; Sat: Saturation; Temp: Temperature. Data are presented as mean \pm SD.* indicate $p<0.05$.

Figure 1: A) drawing of a pregnant doe in the setting of this experiment, rabbits were intubated, received IV and IA line, saturation and temperature monitoring and underwent a median laparotomy that partially exposed the uterus for fetal ultrasound. B) the first two ovarian-end pups in each horn in the bicornuate uterus of the rabbit were randomized to receive ultrasound guided injection with fetal medication (yellow) or saline (black), while the third pup, an untouched littermate served as control. C) Ultrasound image showing the needle injecting the fetal medication in the liver of the pup.

Figure 2: Neurobehavioral outcome on postnatal day 1. A) motor scores. B) sensory scores. Data are presented as individual data points, with mean and SD.

Figure 3: A) Representative image of the hippocampus stained with Hoechst (nuclei) and Neu-N (neurons). B) Neuron density in the 4 investigated regions. Data are presented as individual data points, with mean and SD.

Figure 4: A) Representative image of the hippocampus stained with Hoechst (nuclei) and SY-38 (synapses). B) Integrated density synaptophysin in the 4 investigated regions of interest. Data are presented as individual data points, with mean and SD, * indicates $p<0.05$.

Supplement Figure 1: A) Heart rates of the fetuses in the different groups before and after injection.

B) brain-to-body weight ratios at birth. Data are presented as individual data points, with mean and SD.

Supplement Figure 2: A) Representative image of the hippocampus stained with stained with $\mathrm{KI}-67$ (proliferative cells; black) and Mayer's hematoxylin (nuclei; bleu). B) Proliferation rates in the 4 investigated regions. Data are presented as individual data points, with mean and SD.

This article is protected by copyright. All rights reserved. 


\begin{tabular}{|l|l|l|l|}
\hline & Begin procedure & End procedure & Normal value (range) \\
\hline MAP (mmHg) & $40 \pm 14$ & $50 \pm 15$ & $57(41-72)$ \\
\hline HR (beats/min) & $211 \pm 36$ & $223 \pm 26$ & $237(177-286)$ \\
\hline Sat $\mathbf{O}_{2}(\%)$ & $98 \pm 3$ & $97 \pm 3$ & $97(94-100)$ \\
\hline Temp (' $\mathrm{C})$ & $38.0 \pm 0.8$ & $37.5 \pm 0.8$ & $38(37-39)$ \\
\hline pH & $7.32 \pm 0.08 *$ & $7.40 \pm 0.04$ & $7.44(7.38-7.48)$ \\
\hline pCO $(\mathrm{mmHg})$ & $33 \pm 21$ & $2.4 \pm 9$ & $30.4(23.8-36.8)$ \\
\hline Lactate (mmol/L) & $2.4 \pm 1.1$ & $2.1 \pm 1.3$ & $2.1(0.7-7.2)$ \\
\hline
\end{tabular}


A

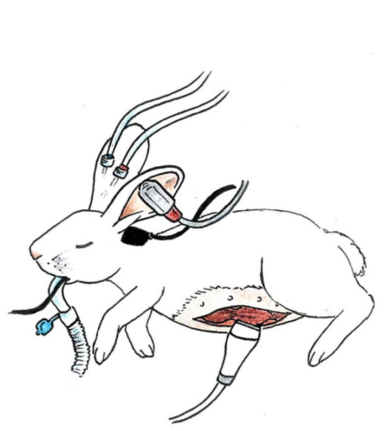

B

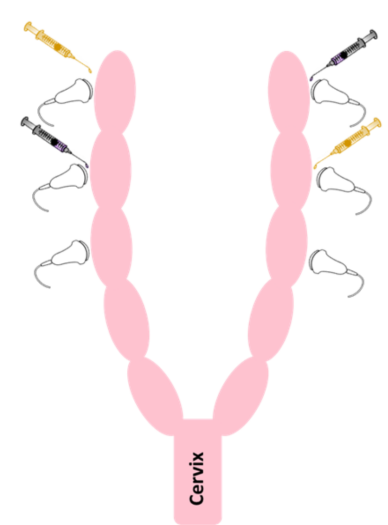

C

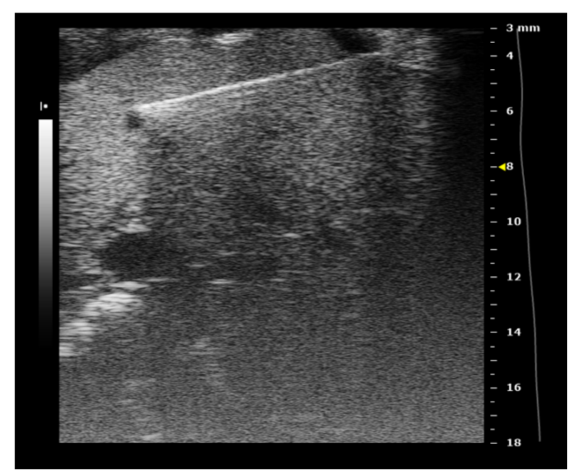

This article is protected by copyright. All rights reserved. 

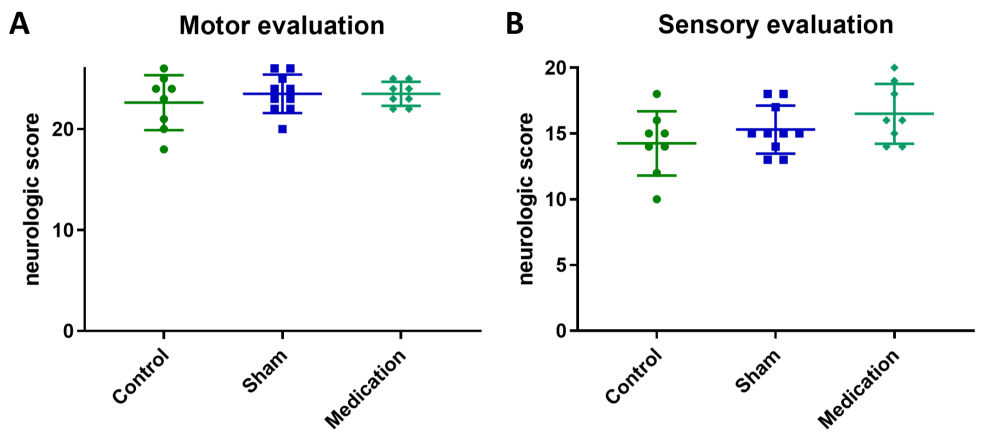

This article is protected by copyright. All rights reserved. 


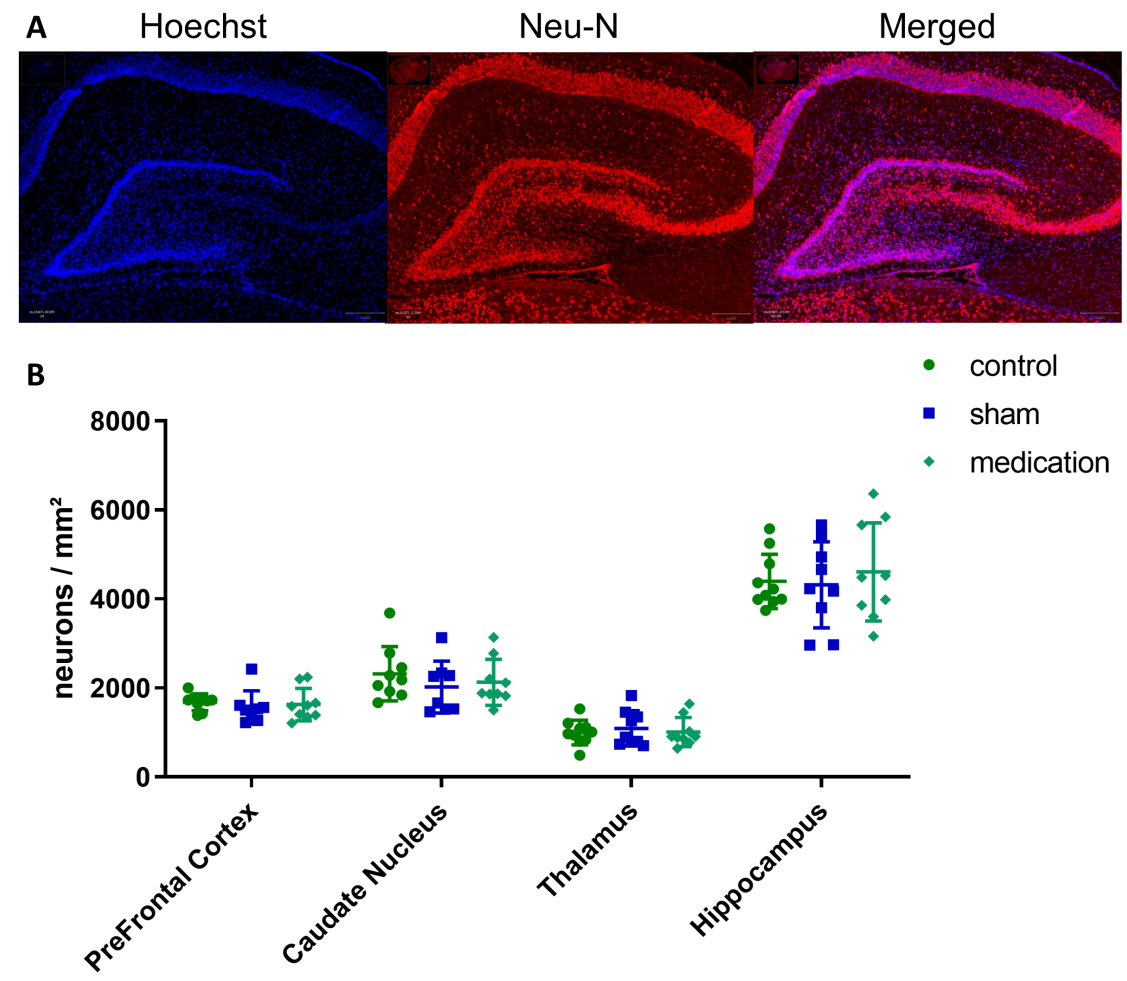

This article is protected by copyright. All rights reserved. 

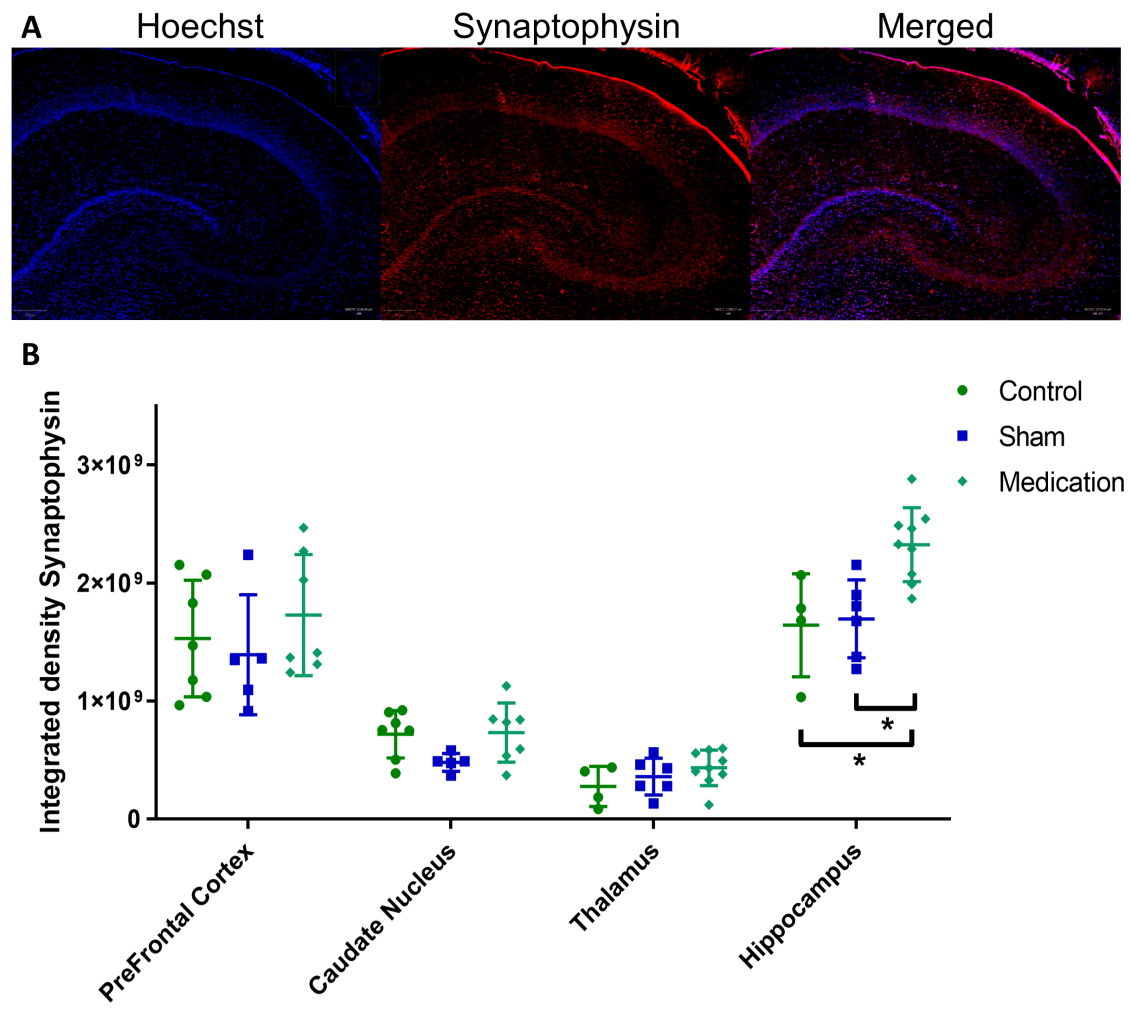

This article is protected by copyright. All rights reserved. 